\title{
High-index-contrast, photonic-band-edge waveguides for tunable time delays
}

\author{
M. L. Povinelli ${ }^{a, b}$, Steven G. Johnson ${ }^{c}$, and J. D. Joannopoulos ${ }^{b}$ \\ ${ }^{a}$ Department of Physics and the Center for Materials Science and Engineering, Massachusetts \\ Institute of Technology, 77 Massachusetts Avenue, Cambridge, MA 02139 \\ ${ }^{b}$ Ginzton Laboratory, Stanford University, Stanford, CA 94306 \\ ${ }^{c}$ Department of Mathematics, Massachusetts Institute of Technology, 77 Massachusetts \\ Avenue, Cambridge, MA 02139
}

\begin{abstract}
We describe the use of high-index-contrast, photonic-crystal wavegides for tunable time delays. The waveguide is designed such that the operating frequency is near a photonic band edge. In this slow light region, a small change in index yields a large change in group velocity, and consequently in time delay. Figures of merit for tunable time delay devices are introduced, including sensitivity, length, and dispersion. We show that a simple quadratic band model is a good predictor of the figures of merit for realistic, 3D, high-index-contrast structures. By cascading two grated waveguides, we can obtain a flat tunable time delay across the operating bandwidth.
\end{abstract}

Keywords: Integrated optical devices, tunable time delays, photonic-crystal waveguides, slow light

\section{INTRODUCTION}

Delaying an optical signal is useful for a number of applications, including optical buffering, signal processing, logic, radio-frequency (RF) photonics, and enhanced nonlinearities. ${ }^{1}$ Several recent research efforts have focussed on replacing relatively bulky fiber-optic delay-line systems ${ }^{2}$ with compact integrated devices. Approaches have included the use of all-pass filters ${ }^{1,3,4}$ and coupled-resonator optical waveguides..$^{5-13}$ Here, we examine an alternate approach to tunable, integrated time delays: slow-light, band-edge photonic-crystal waveguides. Previous work in ideal, 1-D structures has suggested that near a photonic band edge, a large delay tunability can be achieved for a small change in refractive index. ${ }^{14}$ We investigate this slow-light enhancement phenomenon in realistic, three-dimensional structures amenable to on-chip fabrication using established lithographic techniques. In contrast to other work, ${ }^{15}$ our structures are designed to operate in transmission rather than reflection and use uniform rather than chirped gratings. We show that cascading two gratings gives a flat tunable time delay across large operating bandwidths $>100 \mathrm{GHZ}$.

The outline of the paper is as follows. In Sects. 2 and 3 we describe the principle of operation of our device and introduce relevant figures of merit to describe tunable time delays, namely sensitivity, length, and dispersion. We then introduce a quadratic band model to predict the dependence of the figures of merit on distance from the band edge and grating strength in Sect. 4 . The validity of the quadratic band model is demonstrated by explicit comparison to calculations for a realistic, three-dimensional structure in Sect. 5, simplifying device design and optimization. Sect. 6 illustrates how two gratings can be cascaded to compensate for dispersion near the band edge. Using the quadratic band model, we show that nearly-flat time delays can be achieved across the operating bandwidth. Sect. 7 summarizes our results.

E-mail: mpovinel@alum.mit.edu

Tuning the Optical Response of Photonic Bandgap Structures II, edited by Philippe M. Fauchet,

Paul V. Braun, Proceedings of SPIE Vol. 5926 (SPIE, Bellingham, WA, 2005)

0277-786X/05/\$15 - doi: 10.1117/12.617836

Proc. of SPIE Vol. 5926 59260D-1 
(a)

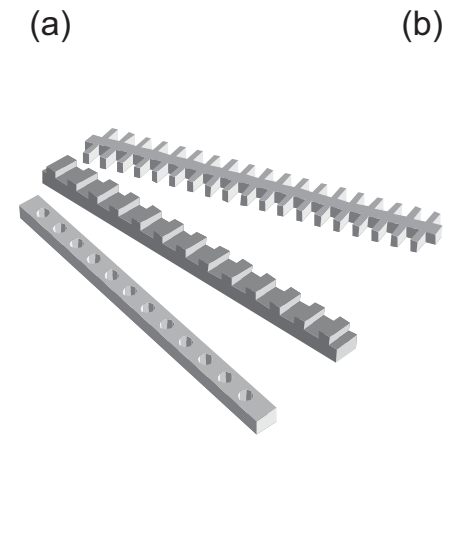

(b)

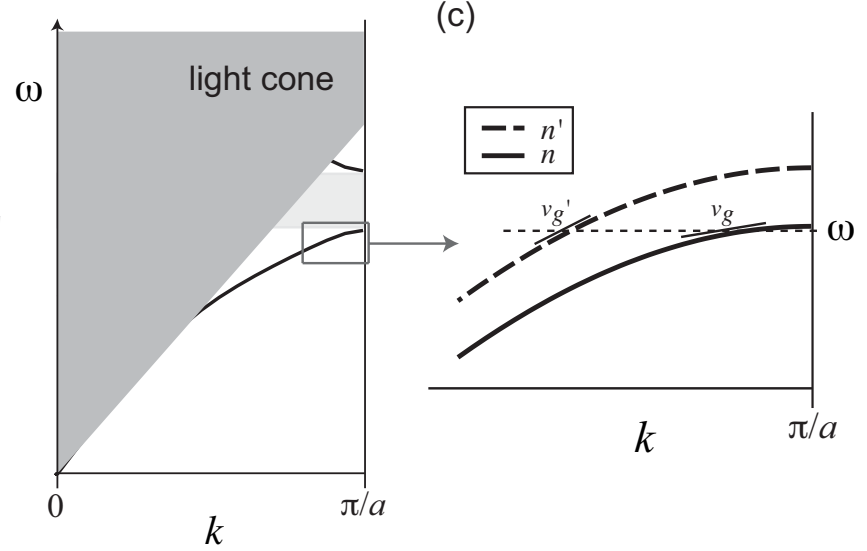

Figure 1. (a) Types of 1-D periodic gratings. (b) Typical band structure of a 1-D periodic grating. The band gap is shaded light gray. (c) Magnified view of the band structure near the band edge, illustrating how a small shift in refractive index can lead to a large shift in group velocity.

\section{PRINCIPLE OF OPERATION}

We begin by describing the principle of operation of our slow-light, band-edge tunable time delay device. A wide variety of 1-, 2-, and 3-D periodic, dielectric structures have dispersion relations with zero group velocity at the edge of the Brillouin zone. ${ }^{16}$ Here we will focus on 1-D periodic gratings with finite width and height, such as those shown in Fig. 1(a), which can be relatively easily integrated on-chip. A grating may be made, for example, by etching teeth in the side of the waveguide, grooves in its top surface, or holes spaced along its length. Such gratings have band structures with the general features shown in Fig. 1(b). The light cone region indicates modes that are extended in either the air or the substrate and is shaded grey. Guided modes of the grating are plotted as solid lines and specified by a frequency $\omega$ and a wave vector $k$ along the waveguide axis. A range of typical 1-D periodic structures exhibit a band gap (shaded light gray) in the guided modes lying below the light line. At the edge of the band gap, the group velocity $d \omega / d k$ of a guided mode goes to zero. Fig. 1(c) shows a magnified view of this region. A small shift in refractive index from $n$ to $n^{\prime}$, induced for example by thermal, optical, or electrical tuning, will cause a small shift in the band. For constant operating frequency, indicated by the horizontal dotted line, a large change in group velocity can result. A large change in group velocity corresponds to a large change in the time required to propagate through a fixed length of grating, and hence a highly-tunable time delay.

Strictly speaking, the use of a band structure to describe the grating is a bulk analyis. For a finite grating length, transmission through the grating is determined not only by the guided modes of an infinite grating, but by Fabry-Perot reflections from the grating ends. However, the magnitude of such reflections can be made arbitrarily small by appropriate tapering of the grating ends, as proved rigorously in Ref. 17 . We thus adopt a bulk analysis to define figures of merit for our device.

\section{FIGURES OF MERIT}

We introduce three figures of merit useful for characterizing any tunable time-delay device.

The first is the sensitivity, a dimensionless figure of merit given by the fractional change in time delay over the fractional change in index:

$$
s \equiv \frac{\Delta \tau / \tau}{\Delta n / n}=\frac{\Delta\left(1 / v_{g}\right)_{\Delta n}}{1 / v_{g}} \frac{1}{\Delta n / n},
$$

where $\tau$ is the time delay, $v_{g}$ is the waveguide group velocity, and $\Delta\left(1 / v_{g}\right)_{\Delta n}=1 / v_{g}^{\prime}-1 / v_{g}$ is the change in group velocity due to a shift from $n$ to $n^{\prime}=n+\Delta n$. $s$ indicates the advantage of the slow-light waveguide structure as compared to a bulk material. For bulk, $\omega=c k / n$ and $s=1$. 
For a fixed fractional index shift $\Delta n / n$ and a desired tunable delay $\Delta \tau$, the required length is given by

$$
L=\left|\frac{\Delta \tau}{\Delta\left(1 / v_{g}\right)_{\Delta n}}\right|=\left|\frac{\Delta \tau}{\Delta n / n} \frac{v_{g}}{s}\right|
$$

Waveguides with low group velocity and high sensitivity allow a shorter tuning region, leading to lower power and ease of integration.

Sensitivity is high near the band edge because of group-velocity dispersion $\left(d^{2} \omega / d k^{2} \neq 0\right)$, but dispersion can also limit the performance of the device. It is useful to define a dispersion figure of merit to measure the amount of pulse spreading relative to the typical size $1 / \Delta \omega$ of temporal features (e.g. pulses or chirp autocorrelations ${ }^{18}$ ), where $\Delta \omega$ is the bandwidth.

$$
d \equiv \frac{\left.\tau\right|_{\omega}-\left.\tau\right|_{\omega-\Delta \omega}}{1 / \Delta \omega}=\frac{L \Delta\left(1 / v_{g}\right)_{\Delta \omega}}{1 / \Delta \omega}
$$

where $\left.\Delta\left(1 / v_{g}\right)_{\Delta \omega} \equiv \frac{1}{v_{g}}\right|_{\omega}-\left.\frac{1}{v_{g}}\right|_{\omega-\Delta \omega}$ is the change in inverse group velocity across the bandwidth. Making use of Eq. 2, $d$ may alternatively be written as

$$
d=|\Delta \tau| \Delta \omega \frac{\Delta\left(1 / v_{g}\right)_{\Delta \omega}}{\left|\Delta\left(1 / v_{g}\right)_{\Delta n}\right|} .
$$

\section{QUADRATIC BAND MODEL}

We next use an approximate model for the dispersion relation near the band edge to yield useful approximations for the figures of merit above. Near the band edge $\omega_{\text {be }}, \omega(k)$ can be approximated by a quadratic curve,

$$
\omega(k)=\omega_{\mathrm{be}}-\alpha(\pi / a-k)^{2},
$$

where $\alpha=-\frac{1}{2} d^{2} \omega /\left.d k^{2}\right|_{\pi / a}$. Large values of $\alpha$ correspond to large curvature of the band structure: the group velocity changes from a large, non-zero value to zero over a small frequency range. Such behavior is characteristic of weak gratings, which have a small band gap. Strong gratings, which have larger band gaps, have small values of $\alpha$.

The shifted band may similarly be written as

$$
\omega^{\prime}(k)=\omega_{\mathrm{be}}+\delta \omega-(\alpha+\delta \alpha)(\pi / a-k)^{2} .
$$

Let $\Delta \omega_{\mathrm{be}} \equiv \omega_{\mathrm{be}}-\omega$. Then

$$
s \approx-\frac{1}{\Delta n / n}\left(1-\frac{1}{\sqrt{\left(1+\delta \omega / \Delta \omega_{\mathrm{be}}\right)(1+\delta \alpha / \alpha)}}\right) .
$$

As the operating frequency approaches the band edge, $|s|$ increases until it reaches a limiting value of $|n / \Delta n|$. Unlike in a bulk medium, $s$ diverges as $\Delta n / n \rightarrow 0$. The divergence in $s$ can be interpreted as a signature of slow-light enhancement of the time-delay tunability, since $s$ increases with decreasing $v_{g}$.

The length is given by

$$
L \approx\left|\frac{2 \Delta \tau \sqrt{\alpha \Delta \omega_{\mathrm{be}}}}{1-\left[\left(1+\delta \omega / \Delta \omega_{\mathrm{be}}\right)(1+\delta \alpha / \alpha)\right]^{-1 / 2}}\right| .
$$

$L$ decreases to zero near the band edge, scaling as $L=2\left|\Delta \tau\left(\alpha \Delta \omega_{\mathrm{be}}\right)^{1 / 2}\right|$ for $\left|\Delta \omega_{\mathrm{be}}\right| \ll|\delta \omega|$. For a given, desired time delay and a fixed distance from the band edge, small $\alpha$ (corresponding to strong gratings) give the shortest device lengths.

The dispersion figure of merit is approximated by

$$
d \approx \frac{L \Delta \omega}{2 \sqrt{\alpha}}\left[\frac{1}{\sqrt{\Delta \omega_{\mathrm{be}}}}-\frac{1}{\sqrt{\Delta \omega_{\mathrm{be}}+\Delta \omega}}\right] .
$$



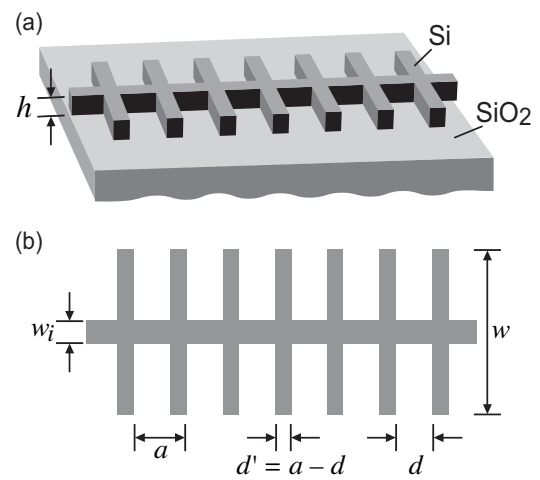

(c)

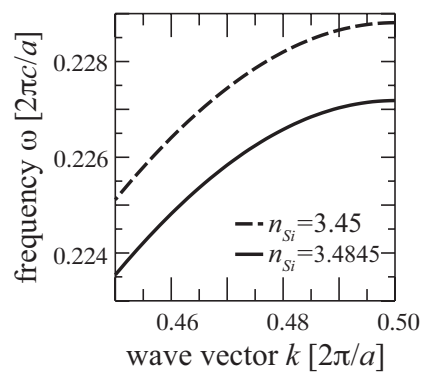

Figure 2. (a) 3-D perspective view of a slow-light grating structure. (b) Top view. (c) Band structure.

Because $L$ scales as $\sqrt{\alpha}$ (up to small corrections that are first order in $\delta \alpha / \alpha \propto \Delta n / n$ ), $d$ is nearly independent of $\alpha$ within the quadratic band model. Approaching the band edge, dispersion increases to a limiting value of $|\Delta \tau| \Delta \omega$. This quantity can be interpreted as a tunable delay-bandwidth product. Specifying an allowable dispersive spread limits the achievable time delay for a given bandwidth. At the band edge, this limit takes the form $|\Delta \tau|<d / \Delta \omega$.

We note that in general, the values of $s$ and $L$ depend on the available tunable index shift, $\Delta n / n$. For this reason, different device designs should be compared using a fixed $\Delta n / n$ value. However, the quadratic band model expressions above show that $L$ is actually independent of the index shift for frequencies sufficiently close to the band edge, though $s$ is not. The dispersion figure of merit $d$ is also independent of the index shift, since it is defined entirely in terms of the unshifted band, for which the operating frequency is closest to the band edge and the dispersive effects are most significant.

\section{NUMERICAL RESULTS}

To explicitly verify the utility of the quadratic band model, we present exact numerical results for the figures of merit for a realistic slow-light structure. We consider the structure shown in Figure 2 (a) and (b), a silicon strip waveguide with grated sidewalls ${ }^{19}$ resting on a silicon-dioxide substrate. Silicon, in particular, has low absorption near $1.55 \mu \mathrm{m}$, high refractive index, and large change in index with temperature; the index varies by $>1 \%$ from 100 to $300{ }^{\circ}{ }^{20}$

Sensitivity, length, and dispersion figures of merit were calculated from the dispersion relation of the structure, which was obtained from fully-vectorial solutions of Maxwell's equations in a plane-wave basis. ${ }^{21}$ Structural parameters were determined by maximizing the size of the first band gap under the constraint that the minimum feature size be at least $0.15 \mu \mathrm{m}$. The parameters are $h=0.26 \mu \mathrm{m}, w_{i}=0.15 \mu \mathrm{m}, a=0.35 \mu \mathrm{m}, d^{\prime}=0.15 \mu \mathrm{m}, d=0.20 \mu \mathrm{m}$, and $w=1.0224 \mu \mathrm{m}$. For $n_{\mathrm{Si}}=3.4845$ and $n_{\mathrm{SiO}_{2}}=1.45$, the first band gap was $23.5 \%$ of the midgap frequency for odd modes with respect to the mirror plane of the structure $(\vec{E}$ mostly parallel to the substrate $)$. For $n_{\mathrm{Si}}=3.4845$, the band edge frequency was calculated to be $\omega_{\mathrm{be}}=0.227182 \pi c / a$ for the lowest band, or $\omega_{\mathrm{be}} / 2 \pi=190 \mathrm{THz}$ $(\lambda=1.54 \mu \mathrm{m})$. For $n_{\mathrm{Si}}^{\prime}=3.45(\Delta n / n=-0.01)$, the band edge shifted to $\omega_{\mathrm{be}}=0.228802 \pi c / a$, or $\omega_{\mathrm{be}} / 2 \pi=200 \mathrm{THz}$ $(\lambda=1.53 \mu \mathrm{m})$. Figure 2 (c) shows the lowest band near the band edge.

Figs. 3 and 4 show the results of the exact numerical calculation (symbols) overlayed on the expressions for $s, L$, and $d$ obtained from the quadratic band model of Eqs. 5, 6, and 7 (solid lines). As can be seen from the figure, the quadratic band model is an excellent predictor of the results within at least $1 \%$ from the band edge. In general, we expect the range of validity of the quadratic band model to decrease as the band gap is decreased. Values of $\delta \omega(0.001622 \pi c / a)$ and $\alpha(1.550 \mathrm{ca} / 2 \pi)$, and $\delta \alpha(0.025 \mathrm{ca} / 2 \pi)$ were obtained from a fit to the data of Fig. 2 (c) at $k=\pi / a$. Figure 3 (a) exhibits the expected increase of $s$ as the operating frequency approaches the band edge (see Eq. 5). Figure 3 (b) exhibits the expected linear scaling of $L$ with the required time delay, $\Delta \tau$ (see Eq. 6). From Eq. 7, we expect $d$ to scale linearly with the tunable delay $\Delta \tau$, as shown in Figure 4 (a). The dependence on bandwidth $\Delta \omega$ is more complex, as shown in Figure 4(b). 
(a)

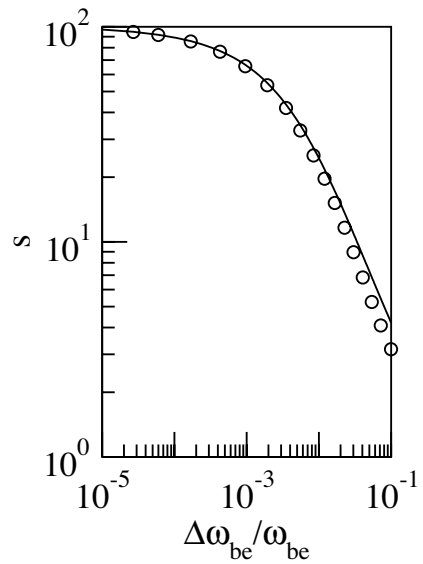

(b)

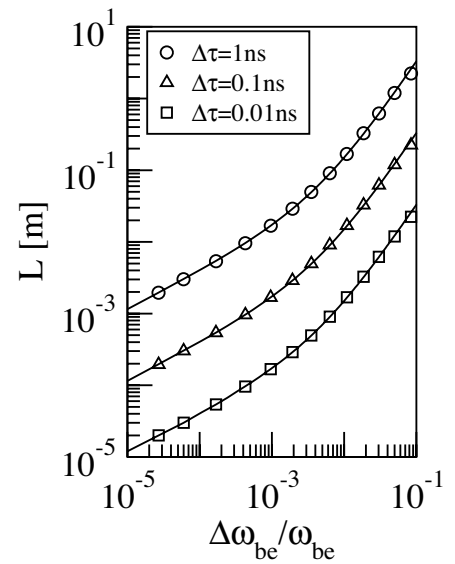

Figure 3. (a) Sensitivity figure of merit for the structure of Fig. 2 as a function of fractional frequency from the band edge. (b) Required length for different amounts of tunable time delay. Symbols/solid lines are exact/quadratic-approximation calculations for a fractional index shift $\Delta n / n=-0.01$.

(a)

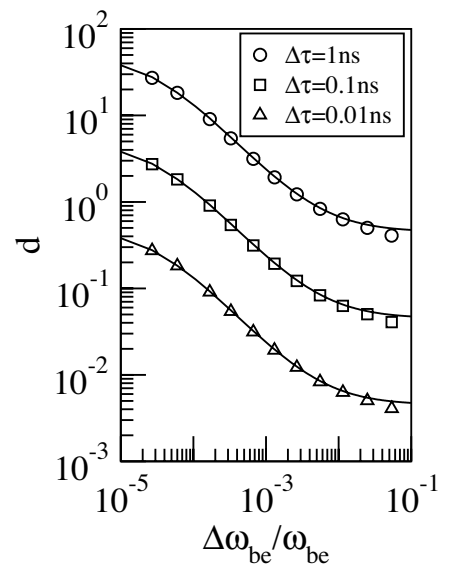

(b)

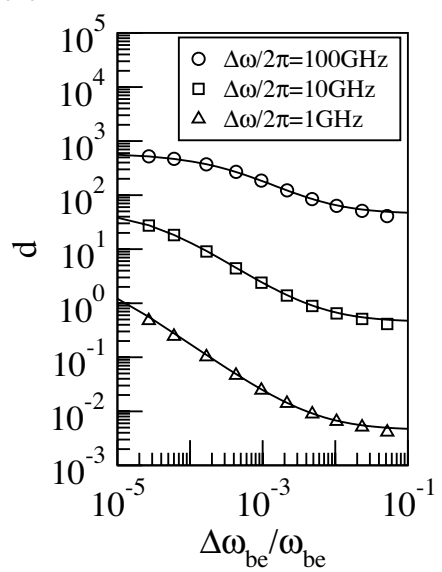

Figure 4. Dispersion figures of merit for the structure of Fig. 2 as a function of fractional frequency from the band edge for (a) fixed bandwidth $\Delta \omega / 2 \pi=10 \mathrm{GHz}$ and (b) fixed time delay $\Delta \tau=1 \mathrm{~ns}$. Symbols/solid lines are exact/quadraticapproximation calculations for a fractional index shift $\Delta n / n=-0.01$.

To illustrate the utility of the figures of merit, let us consider a tunable time delay of $1 \mathrm{~ns}$. Assuming that we can operate at a frequency $2 \%$ away from the band edge, Figure 3 (b) shows that a length of $0.4 \mathrm{~m}$ is required. Simple estimates show that meters of grating can easily be fit onto chips with areas less than $0.1 \mathrm{~cm}^{2}$. For a $1 \mathrm{GHz}$ signal bandwidth, the pulse duration is $\sim 1 \mathrm{~ns}$, and the tunable time delay is comparable to the pulse duration. Meanwhile, the dispersive spread may be obtained from Figure 4 (b) and is less than one-hundredth of the pulse width. For a $10 \mathrm{GHz}$ bandwidth, the pulse width is 10 times shorter, and a tunable delay of $1 \mathrm{~ns}$ corresponds to 10 times the pulse width. However, the dispersive spread is now comparable to the pulse width. Moving toward larger bandwidths and longer time delays suggests the use of dispersion compensation, as discussed in Section 6 below.

\section{DISPERSION COMPENSATION}

Signal dispersion can be compensated by using a sequence of two tapered gratings, as shown in Figure 6 . The light first travels through a grating with period $a_{1}$, where $a_{1}$ and the other structural parameters are chosen to 


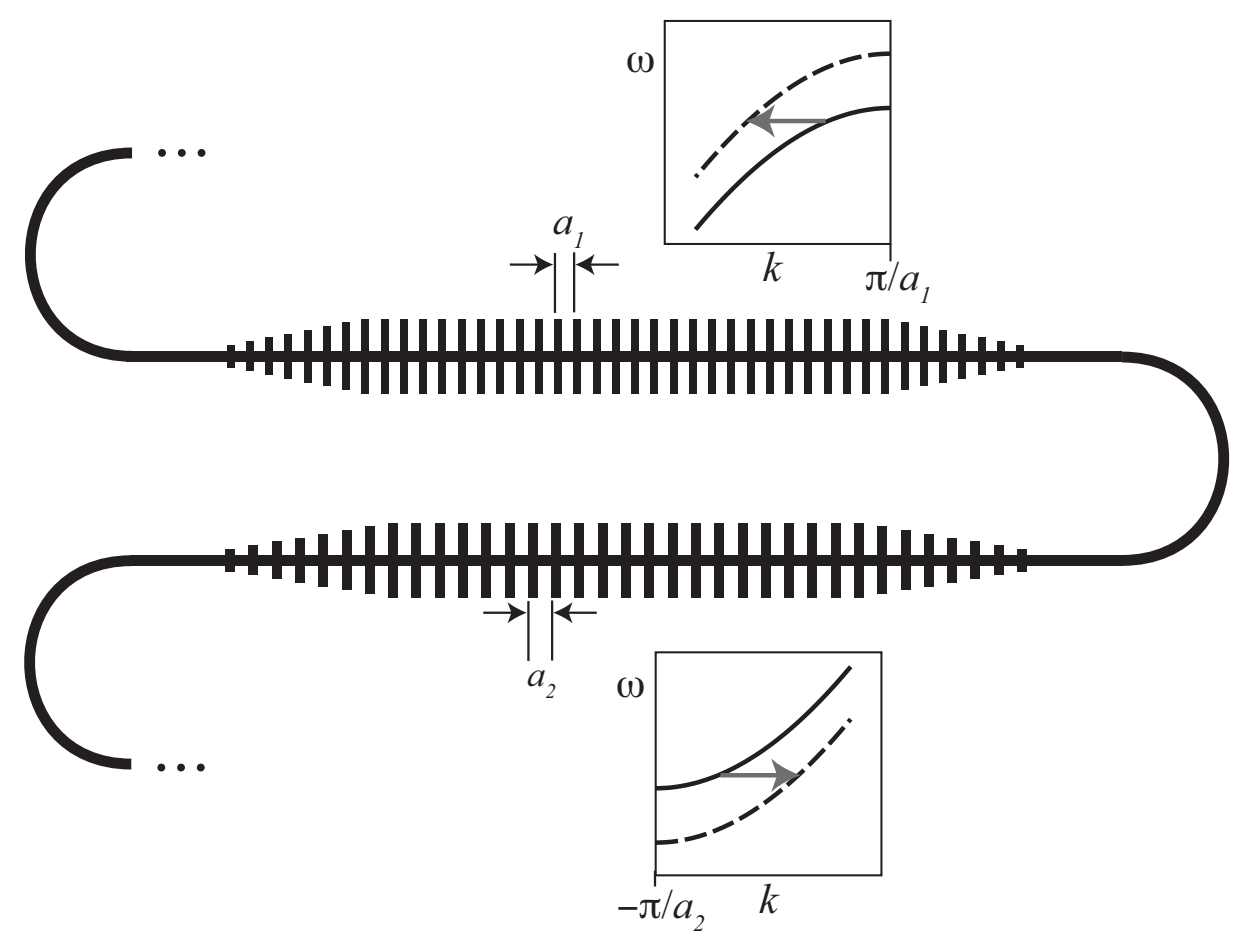

Figure 5. Device design including adiabatically-tapered waveguide segments and dispersion compensation. Insets show the band diagram corresponding to each of the two grating regions.

place the operating frequency at the edge of the lowest band, which has negative curvature. A small decrease in the grating refractive index will reduce the size of the band gap, raising the lowest band and increasing the group velocity. After travelling through the grating, the light enters a second grating with period $a_{2} . a_{2}$ is chosen larger than $a_{1}$, such that the operating frequency falls in the upper band, which has negative curvature. A small decrease in the grating refractive index again reduces the band gap size, shifting the second band down and increasing the group velocity. The net effect of a refractive index shift for the entire device is to increase the total group velocity. Dispersion compensation is a standard practice in optical-fiber communication systems ${ }^{2}$ and Fiber Bragg Gratings. ${ }^{22}$ Similarly here, it is clear that for fixed refractive index (e.g. either the shifted or unshifted band), choosing the dispersion coefficient of the two bands to be equal and opposite at the operating frequency $\omega_{o}$ yields a net zero-group-velocity-dispersion point for grating lengths $L_{1}=L_{2}$. Residual pulse spreading is then governed by weaker, third- and higher-order dispersion.

In the case of a tunable grating, a further quantity of interest is how the tunable delay $\Delta \tau$ varies across the bandwidth. Assume that gratings 1 and 2 are designed to have the same band-edge curvature $(\alpha)$ and to shift by the same amount $\delta \omega$ under tuning. We will center the operating bandwidth midway between the unshifted band edges, such that $\omega_{o}=\left(\omega_{\mathrm{be}, 1}+\omega_{\mathrm{be}, 2}\right) / 2$. Then the dispersion relations for the gratings may be written as

$$
\begin{array}{r}
\omega_{1}(k)=\omega_{o}+\frac{\Delta}{2}-\alpha\left(\pi / a_{1}-k_{1}\right)^{2} \\
\omega_{1}^{\prime}(k)=\omega_{o}+\frac{\Delta}{2}+\delta \omega-\alpha\left(\pi / a_{1}-k_{1}\right)^{2} \\
\omega_{2}(k)=\omega_{o}-\frac{\Delta}{2}+\alpha\left(k_{2}-\pi / a_{2}\right)^{2} \\
\omega_{2}^{\prime}(k)=\omega_{o}-\frac{\Delta}{2}-\delta \omega+\alpha\left(k_{2}-\pi / a_{2}\right)^{2}
\end{array}
$$

where $\Delta / 2 \equiv \omega_{\mathrm{be}, 1}-\omega_{o}=\omega_{o}-\omega_{\mathrm{be}, 2}$. (Small curvature corrections proportional to $\delta \alpha / \alpha<<1$ have been omitted 


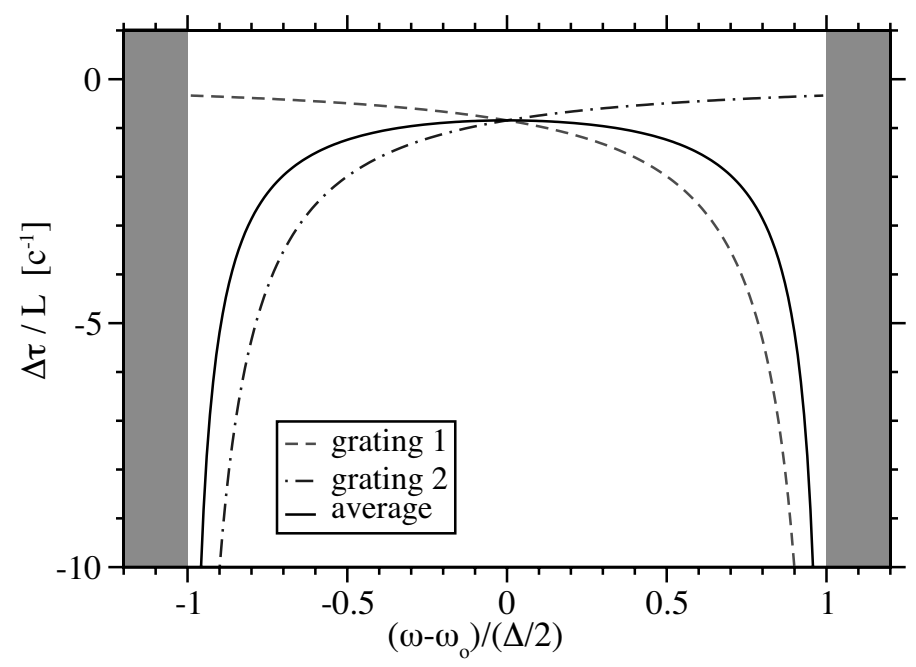

Figure 6. Effect of dispersion compensation on the tunable time delay. Using grating 1 or grating 2 alone causes the tunable delay to vary strongly across the bandwidth. Cascading the two gratings as in Fig. 6 (labelled "average") gives a flat tunable delay in the center of the bandwidth.

for simplicity.) The total tunable time delay is given by

$$
\Delta \tau=\frac{L}{2}\left[\Delta\left(\frac{1}{v_{g, 1}}\right)+\Delta\left(\frac{1}{v_{g, 2}}\right)\right]
$$

where

$$
\begin{aligned}
& \Delta\left(\frac{1}{v_{g, 1}}\right)=\frac{1}{2 \sqrt{\alpha}}\left[\frac{1}{\sqrt{\Delta / 2+\delta \omega-\left(\omega-\omega_{o}\right)}}-\frac{1}{\sqrt{\Delta / 2-\left(\omega-\omega_{o}\right)}}\right] \\
& \Delta\left(\frac{1}{v_{g, 2}}\right)=\frac{1}{2 \sqrt{\alpha}}\left[\frac{1}{\sqrt{\Delta / 2+\delta \omega-\left(\omega_{o}-\omega\right)}}-\frac{1}{\sqrt{\Delta / 2-\left(\omega_{o}-\omega\right)}}\right]
\end{aligned}
$$

and $L=L_{1}+L_{2}$, with $L_{1}=L_{2}$ from the dispersion compensation condition above.

In Fig. 6 we plot the dispersion-compensated tunable delay per unit length, $\Delta \tau / L$ as a function of frequency. Values of $\delta \omega$ and $\alpha$ are as in Section 5 above. We have additionally set $\Delta / 2=0.00454\left(2 \pi c / a_{1}\right)$, corresponding to a $4 \%$ separation of the band edge frequencies of the two gratings. $\Delta \tau / L$ is shown in units of $c$, the speed of light, and the frequency is expressed in dimensionless units as $\left(\omega-\omega_{o}\right) /(\Delta / 2)$. (All time delays are negative due to our convention that the unshifted band has lower group velocity.) The time delay due to grating 1 alone, shown by the dashed line, diverges at the upper end of the frequency range, where $\omega \rightarrow \omega_{\mathrm{be}, 1}$ and $\left(\omega-\omega_{o}\right) /(\Delta / 2)=1$. At lower frequencies, away from the band edge, the time delay approaches a constant. Meanwhile, the time delay due to grating 2 alone, shown by the dashed-dotted line, diverges at the lower end of the frequency range at the band edge of grating 2. The average delay, which enters Eq. 13, diverges at both band edges and is flat across the center of the band, giving a constant time delay. The value is equal to

$$
\Delta \tau / L=\frac{1}{2 \sqrt{\alpha}}\left[\frac{1}{\sqrt{\Delta / 2+\delta \omega}}-\frac{1}{\sqrt{\Delta / 2}}\right] \approx-0.8434 c^{-1} .
$$

In physical units, this corresponds to a tunable delay of $2.811 \mathrm{~ns}$ per meter of device length. The variation in tunable delay per unit length is, moreover, less than $0.03 \%$ across a $100 \mathrm{GHz}$ bandwidth $\left(\left|\left(\omega-\omega_{o}\right) /(\Delta / 2)\right|<\right.$ 0.0128 ) and less than $3 \%$ across a $1 \mathrm{THz}$ bandwidth. 


\section{CONCLUSION}

We have shown that grated waveguides operated in transmission near a photonic band edge enhance the tunability of a time-delay device. The enhancement is due to slow light speeds near the band edge, which result in a large change of group velocity for a small change in index and hence a shorter device length. Explicit numerical calculations for a realistic grating verify that figures of merit for the device are well predicted from a simple quadratic band model. Cascading two adiabatically-tapered gratings both compensates signal dispersion and gives rise to a flat tunable time delay across the operating bandwidth. Optimization, fabrication, and optical characterization of slow-light structures are ongoing. ${ }^{23}$

\section{ACKNOWLEDGMENTS}

The authors thank S. J. Spector, M. W. Geis, R. C. Williamson, T. Lyszczarz, M. Soljačić, and Shanhui Fan for helpful discussions and suggestions. This work was supported by the Defense Advanced Research Projects agency under Air Force Contract \#F19628-00-C-0002. Opinions, interpretations, conclusions, and recommendations are those of the authors, and not necessarily endorsed by the United States Government.

\section{REFERENCES}

1. G. Lenz, B. J. Eggleton, C. K. Madsen, and R. E. Slusher, "Optical delay lines based on optical filters," IEEE J. Quantum Electron. 37, pp. 525-532, Apr. 2001.

2. R. Ramaswami and K. N. Sivarajan, Optical Networks: A Practical Perspective, Academic Press, London, 1998.

3. M. S. Rasras, C. K. Madsen, M. A. Cappuzzo, E. Chen, L. T. Gomez, E. J. Laskowski, A. Griffin, A. WongFoy, A. Gasparyan, A. Kasper, J. LeGrange, and S. S. Patel, "Integrated resonance-enhanced variable delay lines," IEEE Photonics Tech. Lett. 17, pp. 834-6, Apr. 2005.

4. Z. Wang and S. Fan, "Compact all-pass filters in photonic crystals as the building block for high-capacity optical delay lines," Phys. Rev. E 68, p. 066616, December 2003.

5. A. Yariv, Y. Xu, R. K. Lee, and A. Scherer, "Coupled-resonator optical waveguide: a proposal and analysis," Opt. Lett. 24, pp. 711-713, 1999.

6. S. Mookherjea and A. Yariv, "Coupled resonator optical waveguides," IEEE Journal of Selected Topics in Quantum Electronics 8(3), pp. 448-456, 2002.

7. J. Liu, B. Shi, D. Zhao, and X. Wang, "Optical delay in defective photonic bandgap structures," J. Opt. A.: Pure Appl. Opt. 4, pp. 636-639, Oct. 2002.

8. S. Nishikawa, S. Lan, N. Ikeda, Y. Sugimoto, H. Ishikawa, and K. Asakawa, "Optical characterization of photonic crystal delay lines based on one-dimensional coupled defects," Opt. Lett. 27, pp. 2079-2081, Dec. 2004.

9. H. Altug and J. Vučković, "Two-dimensional coupled photonic crystal resonator arrays," Appl. Phys. Lett. 84, pp. 161-163, Jan. 2004.

10. H. Altug and J. Vučković, "Experimental demonstration of the slow group velocity of light in two-dimensional coupled photonic crystal microcavity arrays," Appl. Phys. Lett. 86, p. 111102, 2005.

11. M. F. Yanik and S. Fan, "Stopping light all optically," Phys. Rev. Lett. 92, p. 083901, 2004.

12. M. F. Yanik, W. Suh, Z. Wang, and S. Fan, "Stopping light in a waveguide with an all-optical analog of electromagnetically induced transparency," Phys. Rev. Lett. 93, p. 233903, 2004.

13. D. D. Smith, H. Chang, K. A. Fuller, A. T. Rosenberger, and R. W. Boyd, "Coupled-resonator-induced transparency," Phys. Rev. A 69, p. 063804, 2004.

14. M. Scalora, R. J. Flynn, S. B. Reinhardt, R. L. Fork, M. J. Bloemer, M. D. Tocci, C. M. Bowden, H. S. Ledbetter, J. M. Bendickson, and R. P. Leavitt, "Ultrashort pulse propagation at the photonic band edge: Large tunable group delay with minimal distortion and loss," Phys. Rev. E 54, pp. R1078-1081, Aug. 1996.

15. D. Mori and T. Baba, "Dispersion-contolled optical group delay device by chirped photonic crystal waveguides," Appl. Phys. Lett. 85, pp. 1101-1103, Aug. 2004.

16. J. D. Joannopoulos, R. D. Meade, and J. N. Winn, Photonic Crystals: Molding the Flow of Light, Princeton Univ. Press, 1995. 
17. S. G. Johnson, P. Bienstman, M. A. Skorobogatiy, M. Ibanescu, E. Lidorikis, and J. D. Joannopoulos, "Adiabatic theorem and continuous coupled-mode theory for efficient taper transitions in photonic crystals," Phys. Rev. E 66, p. 066608, Dec. 2002.

18. M. I. Skolnik, Introduction to Radar Systems, ch. 11.5. McGraw-Hill, 1980.

19. J. T. Hastings, M. H. Lim, J. G. Goodberlet, and H. I. Smith, "Optical waveguides with apodized sidewall gratings via spatial-phase locked electron-beam lithography," J. Vac. Sci. Technol. B 20, pp. 2753-2757, Nov/Dec 2002.

20. G. E. Jellison and H. H. Burke, "The temperature depdendence of the refractive index of silicon at elevated temperatures at several laser wavelengths," J. Appl. Phys. 60, pp. 841-843, July 1986.

21. S. G. Johnson and J. D. Joannopoulos, "Block-iterative frequency-domain methods for Maxwell's equations in a planewave basis," Opt. Express 8, pp. 173-190, Jan. 2001.

22. N. M. Litchinitser, B. J. Eggleton, and G. P. Agrawal, "Dispersion of cascaded fiber gratings in WDM lightwave systems," J. Lightwave Tech. 16, pp. 1523-9, Aug. 1998.

23. S. J. Spector, M. W. Geis, and T. Lyszczarz. private communication. 